\title{
Observation of Two Nondispersive Magnetic Excitations in NiO by Resonant Inelastic Soft-X-Ray Scattering
}

\author{
G. Ghiringhelli, ${ }^{1}$ A. Piazzalunga, ${ }^{1}$ C. Dallera, ${ }^{1}$ T. Schmitt, ${ }^{2}$ V. N. Strocov, ${ }^{2}$ J. Schlappa,${ }^{2}$ L. Patthey, ${ }^{2}$ \\ X. Wang, ${ }^{3}$ H. Berger, ${ }^{3}$ and M. Grioni ${ }^{3}$ \\ ${ }^{1}$ CNR-INFM Coherentia and Soft, Dipartimento di Fisica, Politecnico di Milano, piazza Leonardo da Vinci 32, I-20133 Milano, Italy \\ ${ }^{2}$ Swiss Light Source, Paul Scherrer Institut, CH-5232 Villigen PSI, Switzerland \\ ${ }^{3}$ Institut de Physique des Nanostructures, Ecole Polytechnique Fédérale (EPFL), CH-1015 Lausanne, Switzerland
}

(Received 14 July 2008; published 12 January 2009)

\begin{abstract}
We present high resolution ( $\Delta E=120 \mathrm{meV}$ ) resonant inelastic x-ray scattering data measured at the $\mathrm{Ni}$ $L_{3}$ edge $\left(2 p_{3 / 2} \rightarrow 3 d\right)$ on the paradigmatic antiferromagnetic oxide NiO. Spectra reveal clear signatures of magnetic excitations at $\sim 95$ and $\sim 190 \mathrm{meV}$ whose energy seems independent from transferred momentum. These spectral features are well reproduced by a single $\mathrm{Ni}^{2+}$ ion model in an effective exchange field. Within this local model the two magnetic excitations are characterized by a variation of the atomic magnetic moment along the local ordering direction $\left(\Delta S_{\alpha}\right)$ of one and two units. The $\Delta S_{\alpha}=2$ case has different nature from bimagnons observed in optical Raman spectra, for which $\Delta S_{\alpha}=0$.
\end{abstract}

Advanced x-ray techniques have been successfully applied to the study of the structures and ground state properties of magnetically ordered materials. Nonequilibrium properties, on the other hand, remain the exclusive domain of neutrons and of optical spectroscopies. Achieving sensitivity to magnetic excitations, while retaining the characteristic features of $x$-ray resonant techniques, like the chemical and orbital selectivity, is an attractive goal. De Groot, Kuiper, and Sawatzky [1] have pointed out the possibility of measuring local spin-flip excitations by resonant inelastic x-ray scattering (RIXS) performed at the $L_{2,3}$ $(2 p \rightarrow 3 d)$ and $M_{2,3}(3 p \rightarrow 3 d)$ absorption edges of transition metal compounds. Until very recently the experimental confirmation of this prediction had been hampered by practical limitations: the insufficient energy resolution at the $L$ edges and the unfavorable inelastic to elastic intensity ratio at the $M$ edges have inhibited a direct observation of excitations below $500 \mathrm{meV}$, and only indirect signatures of magnetic excitations were found at a larger $(2 \mathrm{eV})$ energy scale [2].

Recent important technical advances have been opening new opportunities for RIXS both in hard [3] and soft $\mathrm{x}$ rays [4]: collective magnetic excitations have been observed in cuprates at the $\mathrm{Cu} K(1 s \rightarrow 4 p)$ [5] and $L_{3}$ edges [6]. We tackle here the case of $\mathrm{NiO}$, a paradigmatic magnetic insulator, antiferromagnetic (AFM) at room temperature. We exploit the unprecedented energy resolution now available at the Swiss Light Source (SLS), where the SAXES spectrometer [4] and the ADRESS beam line [7] have been jointly designed to reach a combined resolving power $>10^{4}$ over the $400-1000 \mathrm{eV}$ energy range. In this Letter we show that in $\mathrm{NiO}$ magnetic excitations can be directly observed by $L_{3}$ RIXS. We have studied their dependence on the incident photon energy and polarization, and on the momentum transfer. We have found that the local model of
Ref. [1] can well reproduce the main features of the experimental results although we cannot exclude the presence in the spectra of dispersing features with much smaller intensity.

During measurements the incident beam was linearly polarized, either within $(H)$ or perpendicular $(V)$ to the horizontal scattering plane, and the scattering angle could be varied between $30^{\circ}$ and $130^{\circ}$ (see inset in Fig. 1). The position and width of the elastic peak, measured on a

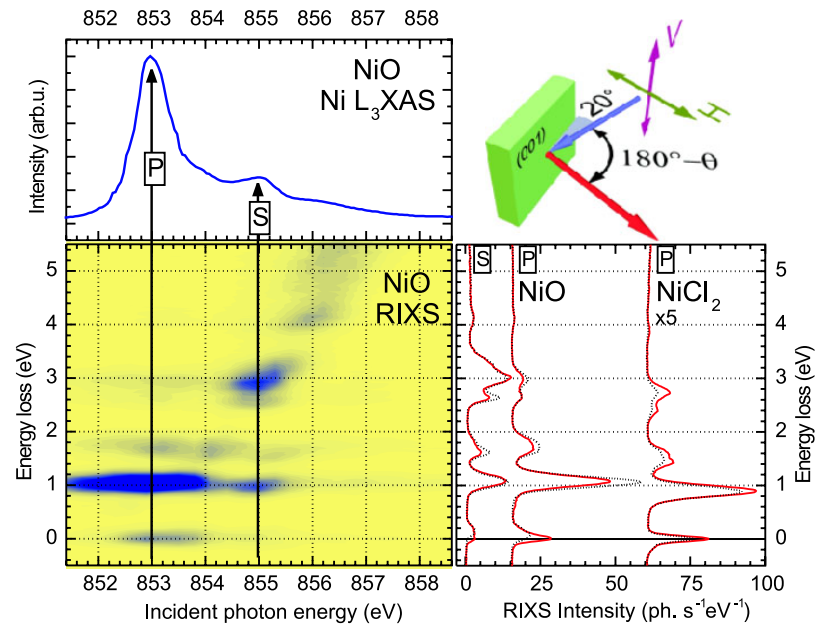

FIG. 1 (color online). Ni $L_{3}$ RIXS spectra measured on $\mathrm{NiO}$, at $90^{\circ}$ scattering angle. The main panel shows, as color map, the RIXS intensity across the $L_{3}$ absorption edge, obtained by combining RIXS spectra excited every $200 \mathrm{meV}$. The XAS spectrum is shown in the top panel; the arrows indicate the main peak $(P)$ and main satellite $(S)$ excitation energies selected for acquiring high quality RIXS spectra. The right panel contains the RIXS spectra of $\mathrm{NiO}$ and $\mathrm{NiCl}_{2}$ : red solid (black dotted) lines are for $V(H)$ polarization, according to the experimental geometry of the inset. 
graphite powder sample, was used to determine the energy loss zero (within $\pm 8 \mathrm{meV}$ ) and the overall instrumentation response (peak full width at half maximum, FWHM, $\Delta E=$ $120 \mathrm{meV}$ at the Ni $L_{3}$ edge, $\sim 853 \mathrm{eV}$ ). The RIXS spectra sampling density is $1 / 12 \mathrm{meV}^{-1}$. All the spectra were obtained by an accumulation of 30 to $60 \mathrm{~min}$ each. X-ray absorption spectra (XAS) were measured in the total electron yield mode by recording the sample current. The single crystal $\mathrm{NiO}$ sample was cut in air along the (100) surface; the pressure in the measurement chamber was $<1 \times 10^{-9}$ mbar. No signs of degradation of the surface were observed over a period of several days. $\mathrm{NiCl}_{2}$ samples were grown by vapor transport. Samples post-cleaved either in air or in vacuum yielded identical spectra. The measurement temperature $T \simeq 300 \mathrm{~K}$ was below the Néel temperature of $\mathrm{NiO}\left(T_{\mathrm{N}}=523 \mathrm{~K}\right)$, but well above that of $\mathrm{NiCl}_{2}(52.3 \mathrm{~K})$ [8].

The color map in Fig. 1 shows an overview of Ni $L_{3}$ RIXS spectra of $\mathrm{NiO}$, where the energy loss is $E=\left(h \nu_{\text {in }}-\right.$ $\left.h \nu_{\text {out }}\right)$. The right panel gives the RIXS spectra of NiO and $\mathrm{NiCl}_{2}$ measured at incident energies $h \nu_{\text {in }}=853.0 \mathrm{eV}$, corresponding to the main peak $(P)$ of XAS (top panel), and $855.0 \mathrm{eV}$ (main satellite, $S$ ). In a purely ionic $\left(\mathrm{Ni}^{2+}\right)$ model, the RIXS process is described as $2 p^{6} 3 d^{8} \rightarrow$ $2 p^{5} 3 d^{9} \rightarrow 2 p^{6} 3 d^{8 *}$, where $2 p^{6} 3 d^{8 *}$ stand for the ground state or an excited state of the $3 d^{8}$ configuration. The $2 p^{5} 3 d^{9}$ intermediate state of RIXS is also the final state of Ni $L_{3}$ XAS. A more realistic description would consider the hybridization of the Ni $3 d$ and ligand $2 p$ orbitals, which yields a noninteger Ni $3 d$ occupation $n_{3 d} \simeq 8.2$ [9], but this is irrelevant for the following analysis of the low-energy excitations. The $\mathrm{NiO}$ spectrum is in good agreement with $L$ and $M$ edge data from the literature [2,10-12], but all spectral features are better defined due to the higher resolution. For both $\mathrm{NiO}$ and $\mathrm{NiCl}_{2}$ at $E \geq 1 \mathrm{eV}$ we recognize the $d d$ excitations of a $\mathrm{Ni}^{2+}\left(d^{8}\right)$ ion in a (nearly) octahedral environment [12]. The data are compatible with a crystal field parameter $10 \mathrm{Dq}$ equal to 1.05 and $0.90 \mathrm{eV}$ for $\mathrm{NiO}$ and $\mathrm{NiCl}_{2}$, respectively $[9,13]$. The similar spectral shape but different energy scale are easy to explain by considering the $\mathrm{Ni}^{2+}$ ion sitting in a nearly octahedral environment and different Ni-ligand distances $(2.08 \AA$ for $\mathrm{Ni}^{2+}-\mathrm{O}^{2-}, 2.43 \AA$ for $\mathrm{Ni}^{2+}{ }_{-} \mathrm{Cl}^{1-}$ ). The effective point charge crystal field is stronger and the Ni $3 d$-ligand $2 p$ overlap is smaller in $\mathrm{NiO}$ than in $\mathrm{NiCl}_{2}$, leading to an overall reduction of the $3 d$ level splitting. A small distortion lowering the local symmetry from $O_{h}$ to $D_{3 d}$ is negligible, and the measured $d d$ losses in RIXS coincide with optical absorption features [14,15].

More remarkable though subtle differences are found close to the (quasi)elastic peak. In $\mathrm{NiCl}_{2}$ the line shape is always nearly Gaussian, almost identical to the elastic peak measured on a graphite sample. By contrast, the quasielastic line of $\mathrm{NiO}$ is broader and clearly asymmetrical due to features in the first $80-100 \mathrm{meV}$. And, mainly at $S$ excita-

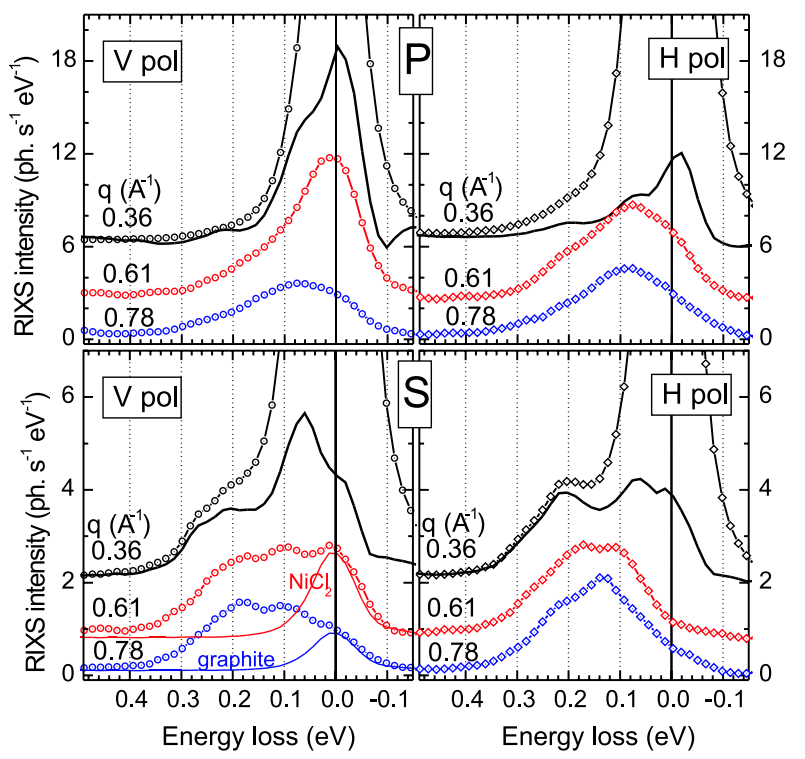

FIG. 2 (color online). The $q$ dependence of the NiO spectra measured at $P$ and $S$ excitation energies, with $V$ and $H$ polarizations. For $q=0.36 \AA^{-1}$ the elastic peak (as measured off resonance on a graphite sample) has been almost totally subtracted in order to highlight the inelastic peaks. For $(S, V)$, also the $\mathrm{NiCl}_{2}$ spectrum (thin red line at $q=0.61 \AA^{-1}$ ) and the elastic peak from graphite (thin blue line at $q=0.78 \AA^{-1}$ ) are shown.

tion, the $\mathrm{NiO}$ spectra are dominated in the first $300 \mathrm{meV}$ by losses totally absent in $\mathrm{NiCl}_{2}$. A close-up of the $\mathrm{NiO}$ data is shown in Fig. 2. With the purpose of detecting possible momentum transfer dependence of the low-energy losses, three different scattering angles $\theta$ have been used, corresponding to three different values of the momentum transferred to the sample in the scattering process $(\vec{q}=$ $\left.\vec{k}_{\text {out }}-\vec{k}_{\text {in }}\right): \quad q\left(50^{\circ}\right)=0.36 \AA^{-1}, \quad q\left(90^{\circ}\right)=0.61 \AA^{-1}$, $\left.q\left(130^{\circ}\right)=0.78 \AA^{-1}\right)$. The AFM zone boundary in the [001], [011], and [111] directions are at $0.75 \AA^{-1}$, $0.80 \AA^{-1}, 0.65 \AA^{-1}$, respectively; in the given experimental setup $\vec{q}$ was at $5^{\circ}$ from [001], at $20^{\circ}$ from [011] and parallel to [011] for $50^{\circ}, 90^{\circ}$, and $130^{\circ}$ scattering, respectively. The intensity of the elastic peak is strongly enhanced towards forward scattering (at $\theta=50^{\circ}$, and especially at $\theta=30^{\circ}$, not shown), so for $\theta=50^{\circ}(q=$ $0.36 \AA^{-1}$ ) we have subtracted the elastic peak (measured off-resonance on graphite) to highlight the inelastic features. All the spectra of Fig. 2 have two common features around 95 and $190 \mathrm{meV}$. For $q=0.36 \AA^{-1}$ the great enhancement of the elastic peak is always accompanied by a $60-70 \mathrm{meV}$ peak. In addition, at $q=0.78 \AA^{-1}$ the $(S, H)$ spectrum shows a pronounced peak at $133 \mathrm{meV}$.

The magnetic nature of low-energy features is evident when comparing $\mathrm{NiO}$ and $\mathrm{NiCl}_{2}$ : the two sample mainly differ in the size of the superexchange interaction, 10 times higher in the oxide than in the chloride, so that magnetic 
excitations in $\mathrm{NiCl}_{2}$ would merge into the elastic peak whereas they can be detectable in $\mathrm{NiO}$ with our energy resolution. Other spectroscopies can also serve to further strengthen our assignment. Most of the losses of Fig. 2 cannot be attributed to single phonons, since their energy exceeds that of transverse $(54 \mathrm{meV})$ and longitudinal (69 meV) optical modes of $\mathrm{NiO}$ [16]. On the other hand, a temperature-dependent spectral feature has been observed at $\sim 0.2 \mathrm{eV}$ in Raman [16] and tunneling experiments [17], and assigned to bimagnon excitations. In infrared absorption spectra a peak at $250 \mathrm{meV}$ has been interpreted as a phonon-assisted bimagnon excitation $[14,18]$. Finally inelastic neutron scattering has mapped the $q$ dispersion of single magnons, revealing an almost isotropic behavior: the Brillouin-zone boundary magnon peak energy is 110 and $120 \mathrm{meV}$ in the [110] and [111] directions, respectively [19]. Although below $140 \mathrm{meV}$ single and multiple phonons might also contribute [20], it is thus reasonable to assign a magnetic origin to the RIXS features in the 100-200 meV range.

The experimental results of Fig. 2 share some remarkable properties with the predictions by de Groot et al. [1]: two peaks dominate the spectra, the second at twice the energy of the first one. Their relative intensities change with excitation energy and photon polarization but not much with the scattering angle; their energy appears to be constant within the experimental uncertainty. We have thus calculated the RIXS spectra in the ionic model with cubic crystal field for all the scattering conditions of our measurements [21] (Fig. 3). $\mathrm{Ni}^{2+}$ ions hold $S=1$ magnetic moment that can change by 1 or 2 units when interacting with scattering photons, which carry spin 1 . So the

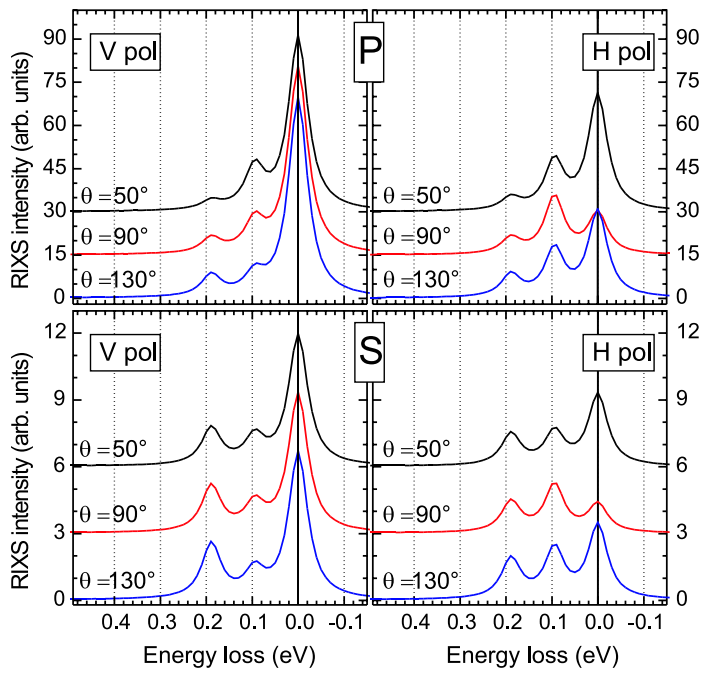

FIG. 3 (color online). Calculated RIXS spectra of a $\mathrm{Ni}^{2+}$ impurity in $O_{h}$ symmetry for excitation at $P$ (bottom) and $S$ (top), and two polarizations of the incident beam. A molecular field corresponding to an effective exchange energy $J^{*}=$ $95 \mathrm{meV}$ is applied. The scattering geometry is the same as in the experimental data of Fig. 2. two peaks correspond to $\Delta S_{\alpha}=1$ and 2 units from the ground state, where $\alpha$ indicates the local magnetic ordering direction. For this reason we can refer to the two peaks as atomic-spin-flip (ASF) excitations, which have local character and are intrinsically different from one- and two- magnon excitations, which have collective nature. Real NiO samples are naturally split into 12 antiferromagnetic domains, which are crystallographically equivalent [19] but different for the RIXS process. We have thus averaged over the spectra calculated for molecular fields along $x, y$, and $z$ directions, and over two perpendicular linear polarizations of the emitted photons because in the experiment the scattered photons polarization was not measured. The strength of the molecular field is given by an effective exchange $J^{*} \simeq 6 J$, where $J$ is the leading interatomic exchange interaction: the two ASF peaks are at $E=J^{*}$ and $E=2 J^{*}$. The calculated spectra confirm and extend the results of Ref. [1]: the $2 J^{*}$ loss is always weak at $P$ but becomes stronger at $S$. We highlight that both ASF peaks correspond to the high spin atomic configuration $(S=1)$. The relative intensities of the three peaks is little sensitive to the scattering angle.

Theory and experiment for $90^{\circ}$ scattering are compared in Fig. 4, where the simulated spectra are those of Fig. 3 convoluted with a Gaussian peak to reach the $120 \mathrm{meV}$ FWHM of the elastic peak. Referring to Figs. 1 and 2, the differences between $\mathrm{NiO}$ and $\mathrm{NiCl}_{2}$ are due to interatomic exchange interaction being 1 order of magnitude smaller in $\mathrm{NiCl}_{2}$ [8] than in $\mathrm{NiO}$. Although even above $T_{\mathrm{N}}$ weak signatures of short-range magnetic correlations are expected around 11 and $22 \mathrm{meV}$, in our $\mathrm{NiCl}_{2}$ data they would be buried under the resolution-limited elastic peak. It is surprising that a simple local scenario already provides such a good description of the data, also in consideration of the actual observation by RIXS of dispersing magnetic excitations in AFM cuprates. In

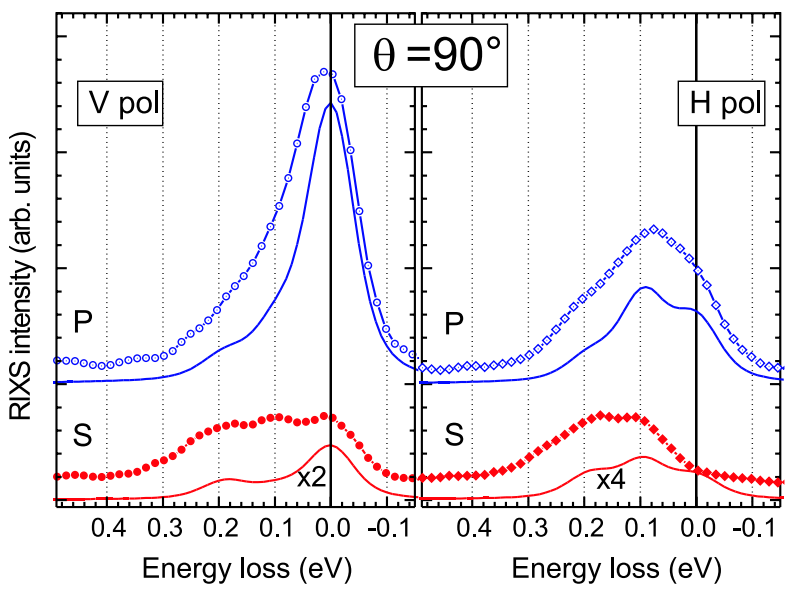

FIG. 4 (color online). Comparison of the experimental RIXS data with the results of the local theory, for an exchange energy $J^{*}=95 \mathrm{meV}$. Experimental spectra are given by symbols, calculations by lines. 
$\mathrm{La}_{2} \mathrm{CuO}_{4}$ the dispersion of a magnetic mode $[5,22]$ has been determined with $L_{3}$ RIXS $[6,23,24]$. Also for cuprates the single ion model predicts one spin-flip peak in $L_{3}$ RIXS, provided that the $\mathrm{Cu}$ atomic moments are not aligned along the $c$ axis [25]. Nevertheless, cuprate spectra appear to be dominated by collective magnetic excitations dispersing up to $380 \mathrm{meV}$, thus exceeding the expected ASF energy (270 meV) [26]. On the contrary in $\mathrm{NiO}$ we find only the two nondispersing ASF peaks: their energy (95 and $190 \mathrm{meV}$ ) is lower than expected from neutrons (110 and $220 \mathrm{meV}$ ) [19], but somehow in agreement with the Raman measurements of bimagnons (193 meV) [14]. Although dispersing features up to $300 \mathrm{meV}$ might be superimposed to the nondispersing peaks, any discussion on them is premature. In fact a variety of collective modes could be in principle excited in $L_{3}$ RIXS, each implying a different change in magnetic moment: $\Delta S_{\alpha}=1$ for a single magnon $\left(E_{\max }=6 J\right), \Delta S_{\alpha}=2$ for a doubleamplitude magnon $\left(E_{\max }=12 J\right), \Delta S_{\alpha}=0$ for 2 magnons $\left(E_{\max } \simeq 12 J\right)$ and 2 double-amplitude magnons $\left(E_{\max } \simeq\right.$ $24 J)$, and other combinations. Thus multiple magnons should be found above $400 \mathrm{meV}$, where intensity in our spectra is negligible, indicating that their cross sections are rather weak.

In conclusion $\mathrm{NiO} L_{3}$ RIXS spectra are characterized by two nondispersing atomic-spin-flip features probably related to single- and double-amplitude magnons but having localized nature. This behavior contrasts with the finding that in 2D AFM cuprates. Although a theory on collective magnetic excitations in $L_{3}$ RIXS is still missing, two parameters are likely to influence cross sections: $J$ (more than 6 time stronger in cuprates than in $\mathrm{NiO}$ ) and dimensionality (cuprates are $2 \mathrm{D}, \mathrm{NiO}$ is $3 \mathrm{D}$ ). On one hand, the bimagnon excitation process is favored by bigger $J$ [24], and thus it should be more probable in cuprates than in $\mathrm{NiO}$. On the other hand in a 3D AFM lattice the bimagnon density of states can become very small when departing from the Brillouin-zone boundaries, leading to negligible bimagnon cross section at low $q$. Although further work at higher resolution will bring a better insight on the dispersion properties of magnetic excitations in AFM compounds as studied by $L_{3}$ RIXS, the data presented here demonstrate that local or collective magnetic excitations carrying $\Delta S_{\alpha}=2$ can now be observed with $\mathrm{x}$ rays. This class of excitations can be studied only involving a spinorbit split intermediate state: $L_{3}$ RIXS is in that quite unique with respect to optical Raman and $K$ edge RIXS (restricted to $\left.\Delta S_{\alpha}=0\right)$, and neutron scattering $\left(\Delta S_{\alpha}=0\right.$ or 1 , actually dominated by the single magnon peak).

This work was performed at the ADRESS beam line of the SLS (PSI) using the SAXES spectrometer developed jointly by Politecnico di Milano, SLS, and EPFL. Technical support by M. Kropf and J. Krempasky and all the SLS staff is gratefully acknowledged. Work at the EPFL is supported by the Swiss NSF and by the NCCR MaNEP. G. G. is indebted to L. Braicovich, J. van den Brink, and L. Ament for illuminating discussions.

[1] F. M. F. de Groot, P. Kuiper, and G. A. Sawatzky, Phys. Rev. B 57, 14584 (1998).

[2] S. G. Chiuzbaian et al., Phys. Rev. Lett. 95, 197402 (2005).

[3] S. Huotari et al., Rev. Sci. Instrum. 77, 053102 (2006).

[4] G. Ghiringhelli et al., Rev. Sci. Instrum. 77, 113108 (2006).

[5] J. P. Hill et al., Phys. Rev. Lett. 100, 097001 (2008).

[6] L. Braicovich et al., arXiv:0807:1140v1.

[7] http://sls.web.psi.ch/view.php/beamlines/adress/index.html

[8] P. A. Lindgard et al., J. Phys. C 8, 1059 (1975).

[9] A. Fujimori and F. Minami, Phys. Rev. B 30, 957 (1984).

[10] H. Ishi et al., J. Phys. Soc. Jpn. 70, 1813 (2001).

[11] M. Magnuson et al., J. Phys. Condens. Matter 14, 3669 (2002).

[12] G. Ghiringhelli et al., J. Phys. Condens. Matter 17, 5397 (2005).

[13] M. G. Brick et al., Physica B (Amsterdam) 371, 43 (2006).

[14] B. Newman and R. M. Chrenko, Phys. Rev. 114, 1507 (1959).

[15] J. Ackerman et al., Inorg. Chem. 11, 3118 (1972).

[16] R. E. Dietz et al., Phys. Rev. B 4, 2302 (1971).

[17] D. C. Tsui et al., Phys. Rev. Lett. 27, 1729 (1971).

[18] J. Lorenzana and G. A. Sawatzky, Phys. Rev. B 52, 9576 (1995).

[19] M. T. Hutchings and E. J. Samuelsen, Phys. Rev. B 6, 3447 (1972).

[20] For $q=0.36 \AA^{-1}\left(0.78 \AA^{-1}\right)$ peaks at $\sim 70 \mathrm{meV}$ $(\sim 135 \mathrm{meV})$ can be assigned to single (double) LO phonons. As dispersing acoustic modes lie below $40 \mathrm{meV}$ and optical modes show very reduced dispersion, the $70 \mathrm{meV}$ and $135 \mathrm{meV}$ peaks at 0.36 and $0.78 \AA^{-1}$ cannot belong to the same phonon branch.

[21] $\mathrm{Ni}^{2+}$ ion in $O_{h}$ symmetry $(10 D q=1.05 \mathrm{eV})$, plus an effective interatomic magnetic interaction $\left(J^{*}\right)$. We have used the MISSING package available at http://www.esrf.fr.

[22] F. H. Vernay M. J. P. Gingras, and T. P. Devereaux, Phys. Rev. B 75, 020403(R) (2007).

[23] J. van den Brink, Europhys. Lett. 80, 47003 (2007).

[24] F. Forte L. J. P. Ament, and J. van den Brink, Phys. Rev. B 77, 134428 (2008).

[25] G. Ghiringhelli et al. (unpublished).

[26] Considering a leading interatomic exchange interaction $J$ with $N$ neighbors having spin $S$, the ASF peaks are expected at $E(\Delta S)=N S J \Delta S$ : for cuprates $(S=1 / 2, J=$ $135 \mathrm{meV}, N=4, \Delta S=1$ ) one peak around $270 \mathrm{meV}$, for $\mathrm{NiO}(S=1, J=20 \mathrm{meV}, N=6, \Delta S=1,2)$ two peaks at 120 and $240 \mathrm{meV}$. 